\title{
Screening Canine Microbiota for Extended Spectrum Beta-Lactamases (ESBLs) Using E-Test
}

\author{
N. Mohammad Sharif ${ }^{*}$, B. Sreedevi ${ }^{1}$, R.K. Chaitanya ${ }^{1}$ and Ch. Srilatha ${ }^{2}$ \\ ${ }^{1}$ Department of Veterinary Microbiology, ${ }^{2}$ Department of Veterinary Pathology, College of \\ Veterinary Science, Sri Venkateswara Veterinary University, Tirupati, Andhra Pradesh, India \\ *Corresponding author
}

\section{A B S T R A C T}

Extended spectrum beta-lactamase (ESBL) production by animal pathogens is an Keywords E. coli, ESBL, Otitis,

Pseudomonas, Proteus, Pyometra, Staphylococcus.

Article Info

Accepted:

23 August 2017 Available Online: 10 September 2017 emerging problem world-wide. E-test was performed for the detection of ESBL production in microbiota isolated from pyometra and otitis clinical cases of dogs presented to Veterinary clinics in Andhra Pradesh. Microbiota recovered from pyometra pus samples includes Escherichia coli (7, 46.6\%), Staphylococcus (11, $73.3 \%$ ) and Pseudomonas (12, 80\%) species. Microbiota recovered from otitis pus samples includes Pseudomonas $(8,53.3 \%)$, Proteus vulgaris $(9,60 \%)$ and Staphylococcus $(15,100 \%)$ species. Of the seven E. coli isolates recovered, three (43\%) isolates were positive for simultaneous production of ESBL and Amp C, two $(28.5 \%)$ isolates were ESBL negative and two (28.5) isolates gave nonconclusive results. Of the 20 Pseudomonas isolates, 16 (80\%) isolates were ESBL negative and four (20\%) isolates gave non-conclusive results. All the 26 Staphylococcus and nine Proteus isolates were found negative for ESBL production.

\section{Introduction}

The extended spectrum beta-lactamases (ESBLs) are the bacterial enzymes that inactivate third-generation cephalosporins, penicillins and monobactams (Bush and Jacoby, 2010). ESBLs are widely detected in various human medical institutions and less frequently from the bacterial population circulating in animals (Carattoli, 2008). Various phenotypic methods used for the detection of ESBLs include combination disc method (CDM), double disc synergy test (DDST), inhibitor-potentiated disc diffusion (IPDD), three-dimensional (3D) test, E-test (Epsilometer gradient test), commercial automated systems like Phoenix, Vitek 2, MicroScan and Sensititre (Drieux et al., 2008 and Sharif et al., 2017). All these methods are based on the principle of antagonism of the action of ESBLs by beta-lactamase inhibitors like clavulanic acid (Drieux et al., 2008). Epsilometer or E-test uses thin E-strips with minimum inhibitory concentration (MIC) scale in $\mu \mathrm{g} / \mathrm{mL}$ and two predefined antibiotic gradients (a gradient of cephalosporin alone at one end and the same gradient with a constant concentration of clavulanic acid at other end) (Drieux et al., 2008). They are intended to confirm the presence of clavulanic acid 
inhibitable ESBL enzymes in various bacterial species (Prabha et al., 2016).

The aim of this study is to screen the microbiota isolated from clinical cases of pyometra and otitis in dogs for ESBL production using E-test method.

\section{Materials and Methods}

\section{Sample collection}

Pus swab samples from clinical cases of otitis $(n=15)$ and pyometra $(n=15)$ were collected from dogs presented to Teaching Veterinary Clinical Complex (TVCC) of College of Veterinary Science (Tirupati), NTR College of Veterinary Science (Gannavaram) and Veterinary Polyclinic (Ongole), Andhra Pradesh, India. All the samples were transported on ice to the laboratory immediately after collection.

\section{Isolation and identification of microbiota}

Pus swab samples were transferred to nutrient broth and incubated aerobically at $37^{\circ} \mathrm{C}$ for 24 hours. Enriched samples were streaked on nutrient agar and mannitol salt agar plates, incubated at $37^{\circ} \mathrm{C}$ and the colonies were tentatively identified based on the colonial morphology, swarming phenomenon, pigment production, Gram's staining and sub-cultured on MacConkey agar. Lactose fermenting pink colonies on MacConkey agar were further streaked onto EMB agar plates. Non-lactose fermenting pale colonies on MacConkey agar were further streaked onto XLD agar plates. Identification of the bacterial isolates to the genus/species level was done using conventional biochemical tests (Sneath and Holt, 2001).

\section{E-test method}

The determination of MIC of cefotaxime and cefotaxime/clavulanic acid for the bacterial strains isolated in the present study was performed using E-test strips (Hi-Media, Mumbai, India) (CTX / CTX+) containing a fixed concentration gradient of cefotaxime alone (CTX, $0.25-16 \mu \mathrm{g} / \mathrm{mL}$ ) at one end of the strip and cefotaxime with clavulanic acid (CTX+, $0.016-1.0 \mu \mathrm{g} / \mathrm{mL})$ on the other end. E-test strips were applied aseptically on Mueller-Hinton agar plates inoculated with pure culture of bacterial isolates, by pressing it with sterile forceps along the whole length of the antibiotic gradient ensuring the complete contact with agar surface. Results were recorded after $18-24 \mathrm{~h}$ of incubation at $37^{\circ} \mathrm{C}$. Inhibition of growth presents as a spherical formation or inhibition ellipse. The MIC is read as the point where the inhibition ellipse intersects the edge of the E-test strip.

The presence of an ESBL was confirmed if there is an 8 fold reduction (MIC ratio of CTX and CTX+ $\geq 8$ ) in the MIC of cefotaxime in presence of clavulanic acid (i.e., MIC value of cefotaxime reduced by more than three doubling dilution steps in the presence of clavulanate) (Drieux et al., 2008). E. coli (ATCC 25922) and Klebsiella pneumoniae (ATCC 700603) were used as negative and positive controls, respectively.

\section{Results and Discussion}

Microbiota isolated from pyometra pus samples includes Escherichia coli $(7,46.6 \%)$, Staphylococcus (11, 73.3\%) and Pseudomomas $(12,80 \%)$ species. In a study by Bassessar et al., (2013) from Madhya Pradesh, E. coli was isolated predominantly (50\%) from canine pyometra cases followed by Salmonella (20\%), Staphylococcus (20\%) and Pseudomonas (10\%) species. Coggan et al., (2008) from Brazil reported $74.1 \%$ incidence of $E$. coli in canine pyometra cases. Microbiota isolated from otitis pus samples includes Pseudomonas (8, 53.3\%), Proteus vulgaris $(9,60 \%)$ and Staphylococcus (15, $100 \%$ ) species. Staphylococcus was by far 
most common bacteria isolated from ear canal of dogs with otitis by many workers (Dixit et al., 2011 and Petrov et al., 2013). Dixit et al., (2011) observed 37.1, 11.4 and $11.4 \%$ incidence of Staphylococcus, Proteus and Pseudomonas species respectively, from otitis cases of dogs.

E-test was performed for the detection of ESBL production in microbiota isolated in the present study (Fig. 1). Interpretation of results was done as per the guidelines of Clinical and Laboratory Standards Institute (CLSI) (Table 1). Among the seven E. coli isolates, three (43\%) isolates were found positive for simultaneous production of ESBL and AmpC (no zone is obtained for CTX and zone obtained in CTX+), two (28.5\%) isolates were found ESBL negative (MIC of CTX $<0.5$ and the ratio of the MIC value of CTX / CTX+ is less than or equal to 8) and the remaining two (28.5) isolates gave non-conclusive results (no zone of inhibition on either side). Resistance in these isolates may be due to mechanisms other than ESBLs such as metallo beta-lactamases and porins (that are not inhibited by beta-lactamase inhibitors) which have to be further investigated before reporting (O'Keefe et al., 2010). The coexistence of both ESBL and AmpC betalactamases could be due to dissemination of plasmid mediated AmpC beta-lactamases among Gram negative bacteria (O'Keefe et al., 2010). The three E. coli isolates found positive for concurrent production of ESBL and AmpC enzymes in the present study were recovered from pus swabs of pyometra cases.

Fig.1 (A) \& (B). E-test ESBL negative result with MIC ratio of (CTX): $(\mathrm{CTX}+)<8$; (C). E-test result showing production of both ESBL and AmpC enzymes (No zone is obtained for CTX and zone obtained in $\mathrm{CTX}+$ ); (D). E-test non-conclusive result (No zone of inhibition on either side).

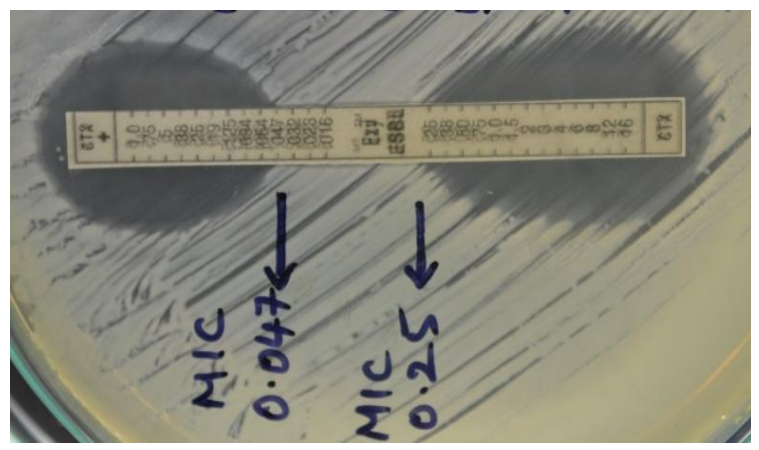

(A)

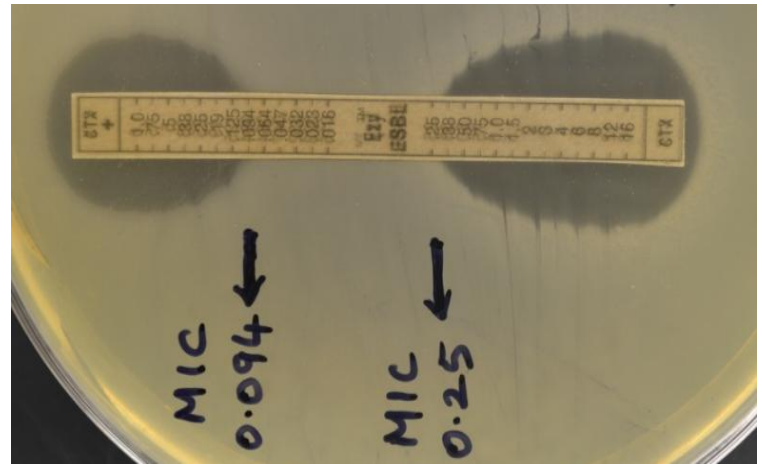

(B)

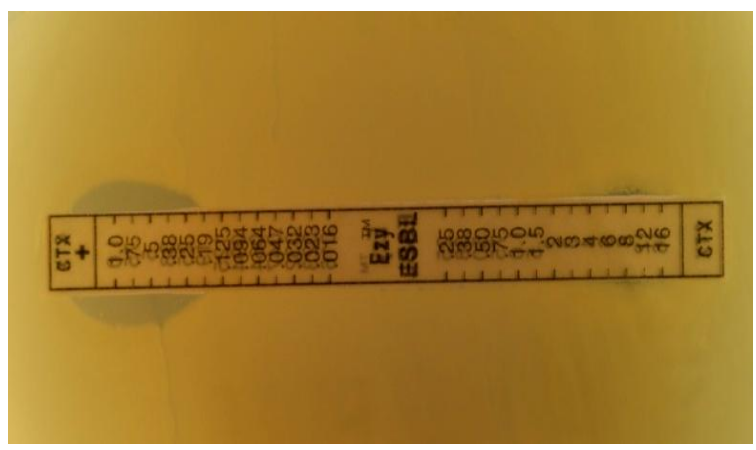

(C)

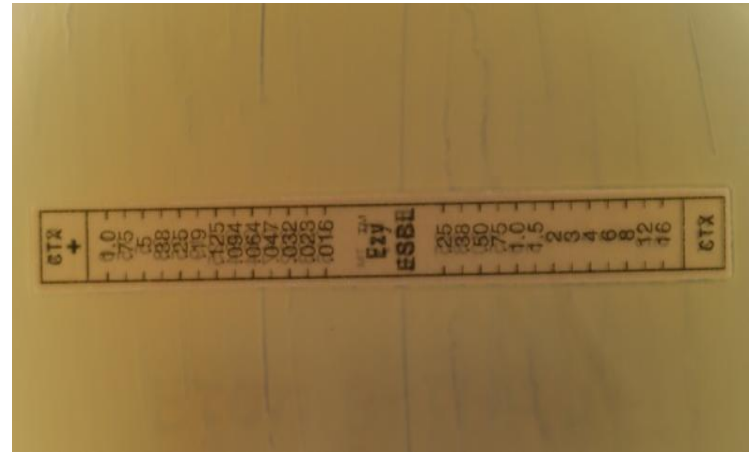

(D) 
Table.1 Interpretation of ESBL E-test results

\begin{tabular}{|l|l|l|}
\hline S. No. & \multicolumn{1}{|c|}{ Result } & \multicolumn{1}{|c|}{ Interpretation } \\
\hline 1. & $\begin{array}{l}\text { CTX } \geq 0.5 \text { and the ratio of the MIC value of } \\
(\text { CTX):(CTX+) is more than } 8\end{array}$ & ESBL positive \\
\hline 2. & $\begin{array}{l}\text { Phantom zone just below the lowest } \\
\text { concentration of CTX+ gradient or a } \\
\text { deformation zone of the CTX inhibition ellipse } \\
\text { at the tapering end }\end{array}$ & ESBL positive \\
\hline 3. & $\begin{array}{l}\text { No zone is obtained for CTX and zone } \\
\text { obtained in CTX+ }\end{array}$ & Production of both ESBL and AmpC \\
\hline 4. & $\begin{array}{l}\text { CTX }<0.5 \text { and the ratio of the MIC value of } \\
(\text { CTX):(CTX+) is less than or equal to } 8\end{array}$ & ESBL negative \\
\hline 5. & No zone of inhibition on either side & Non-conclusive. \\
\hline
\end{tabular}

Among the 20 Pseudomonas isolates, 16 $(80 \%)$ isolates were found ESBL negative $(\mathrm{CTX}<0.5$ and CTX / CTX $+\leq 8)$ and the remaining four $(20 \%)$ isolates gave nonconclusive results (no zone of inhibition on either side). All the 26 Staphylococcus and nine Proteus isolates recovered in the present study were found negative for ESBL production $(\mathrm{CTX}<0.5$ and $\mathrm{CTX} / \mathrm{CTX}+\leq$ 8). O'Keefe et al., (2010) from United States reported detection of ESBL production in 11 (18.3\%) out of $60 \mathrm{E}$. coli isolates recovered from urinary tract infections in dogs using Etest. Tamang et al., (2012) observed a positive confirmatory test for the production of ESBLs in 12 out of 18 cefotaxime resistant $E$. coli isolates recovered from stray dogs in Korea. Huber et al., (2013) from Switzerland, reported $8.4 \%$ incidence of ESBL phenotype among E. coli isolated from dogs with urogenital infections. The sensitivity of E-test for the detection of ESBLs has been shown to vary from 94-100\% (Drieux et al., 2008 and Prabha et al., 2016).

This study provides the first report of detection of concurrent production of both ESBL and AmpC enzymes in E. coli isolates $(43 \%)$ recovered from canine pyometra cases in India using E-test. The presence of an ESBL-producing organism in a clinical infection can result in treatment failure if extended spectrum cephalosporins and monobactams are used in the therapeutic regimens. It emphasize the clinical importance of accurate and early detection of resistant strains in companion animals in order to select the appropriate therapeutic options.

\section{Acknowledgements}

This work was supported by facilities and funds from Sri Venkateswara Veterinary University, Tirupati, Andhra Pradesh.

\section{References}

Bassessar, V., Verma, Y. and Swamy, M. 2013. Antibiogram of bacterial species isolated from canine pyometra. Vet. World., 6(8), 546-549.

Bush, K., and Jacoby, G.A. 2010. Updated functional classification of $\beta$ lactamases. Antimicrob. Agents Chemother., 54, 969-976.

Carattoli, A., 2008. Animal reservoirs for extended spectrum $\beta$ - lactamase producers. Clin. Microbiol. Infect., 14, 117-123. 
Coggan, J.A., Melville, P.A., Oliveira, C.M.D., Faustino, M., Moreno, A.M. and Benites, N.R. (2008). Microbiological and histopathological aspects of canine pyometra. Braz. J. Microbiol., 39(3), 477-483.

Dixit, A.A., Rao, M.L.V., Sharma, V. and Roy, K. 2011. Microbiological study in canine otitis externa. Indian J. Field Vet., 6(4), 47-48.

Drieux, L., Brossier, F., Sougakoff, W. and Jarlier, V. 2008. Phenotypic detection of extended- spectrum $\quad \beta$ - lactamase production in Enterobacteriaceae: review and bench guide. Clin. Microbiol. Infect. 14: 90-103.

Huber, H., Zweifel, C., Wittenbrink, M.M. and Stephan, R. 2013. ESBL-producing uropathogenic Escherichia coli isolated from dogs and cats in Switzerland. Vet Microbiol., 162(2), 992-996.

O'Keefe, A., Hutton, T.A., Schifferli, D.M. and Rankin, S.C., 2010. First detection of CTX-M and SHV extended-spectrum $\beta$-lactamases in Escherichia coli urinary tract isolates from dogs and cats in the United States. Antimicrob. Agents Chemother., 54(8), 3489-3492.

Petrov, V., Mihaylov, G., Tsachev, I., Zhelev, G., Marutsov, P. and Koev, K. 2013.
Otitis externa in dogs: microbiology and antimicrobial susceptibility. Revue Med. Vet., 164(1), 18-22.

Prabha, R., Easow, J.M. and Swapna, M. 2016. Phenotypic detection of Extended Spectrum Beta-Lactamase producing uropathogens using DDST, PCT, Chrom agar and E-test-A comparitive study. Int. J. Curr. Microbiol. App. Sci., 5(4), 565-577.

Sharif, N.M., Sreedevi, B. and Chaitanya, R.K. 2017. Occurrence of beta-lactam resistant Escherichia coli among clinical cases of livestock in Andhra Pradesh. International Journal of Science, Environment and Technology, 6: 1608-1615.

Sneath, P.H.A., and Holt, J.G. 2001. Bergey's manual of systematic bacteriology, $2^{\text {nd }}$ edition. A waverly company, Williams \& Wilkins, Springer-Verlag, NY, USA.

Tamang, M.D., Nam, H.M., Jang, G.C., Kim, S.R., Chae, M.H., Jung, S.C., Byun, J.W., Park, Y.H. and Lim, S.K. 2012. Molecular characterization of extendedspectrum $\beta$-lactamase and plasmid mediated AmpC $\beta$-lactamase-producing Escherichia coli isolated from stray dogs from Korea. Antimicrob. Agents Chemother, pp.AAC-05598.

\section{How to cite this article:}

Mohammad Sharif, N., B. Sreedevi, R.K. Chaitanya and Srilatha, Ch. 2017. Screening Canine Microbiota for Extended Spectrum Beta-Lactamases (ESBLs) Using E-Test. Int.J.Curr.Microbiol.App.Sci. 6(9): 2442-2446. doi: https://doi.org/10.20546/ijcmas.2017.609.300 